Mens

revue d'histoire intellectuelle de l'Amérique française

\title{
Index des volumes I à V de Mens (automne 2000 - printemps
} 2005)

\section{Yves Bégin}

Volume 5, numéro 2, printemps 2005

URI : https://id.erudit.org/iderudit/1024373ar

DOI : https://doi.org/10.7202/1024373ar

Aller au sommaire du numéro

Éditeur(s)

Centre de recherche en civilisation canadienne-française

ISSN

1492-8647 (imprimé)

1927-9299 (numérique)

Découvrir la revue

Citer ce document

Bégin, Y. (2005). Index des volumes I à V de Mens (automne 2000 - printemps

2005). Mens, 5(2), 583-591. https://doi.org/10.7202/1024373ar d'utilisation que vous pouvez consulter en ligne.

https://apropos.erudit.org/fr/usagers/politique-dutilisation/ 
INDEX

\section{$M E N S$ \\ INDEX DES VOLUMES I À V \\ (AUTOMNE 2000 - PRINTEMPS 2005)}

Compilé par

Yves Bégin

Département d'histoire

Université Laval

\section{INDEX DES AUTEURS}

Abréviations :

(ar) article

(éc) édition critique

(bib) bibliographie

(nc) note critique

(nr) note de recherche

(cr) compte rendu

(pers) perspective

Balthazar, Louis : V,1 (nc : 151-161)

Beausoleil, Maude : I, 2 (cr : 174180)

Bédard, Éric : III, 1 (pers : 9-26); $\mathrm{V}, 2$ (cr: 525-531)

Bégin, Yves : I, 1 (cr : 78-80) ; I, 2 (éc : 163-169) ; II, 2 (ar : 155-192)

Béland, Caroline : II, 2 (ar : 233-259)
Bélanger, Damien-Claude : I, 1 (ar : 7-36) ; I, 1 (éc : 69-74) ; I, 2 (bib : 189-223) ; II, 1 (nc : 105112) ; III, 1 (cr: 97-102) ; III, 2 (cr : 288-294) ; IV, 1 (cr : 119-124) Bérubé, Harold : I, 2 (cr : 184-187) ; II, 2 (cr : 271-277)

Bock, Michel : IV, 1 (cr : 136-144) 
Boily, Frédéric : I, 2 (ar : 141-161); IV, 1 (cr : 125-130) ; V, 2 (cr : 502508)

Bourbeau, Amélie : V, 1 (cr : 176181)

Chevrier, Marc : V, 1 (cr : 163-166)

Clermont-Legros, Jean-Francis : II, 2 (bib : 279-297)

Cook, Ramsay : I, 2 (ar : 97-113) ; IV, 2 (nc : 309-319)

Côté, Sébastien : IV, 1 (ar : 39-67)

Coupal, Sophie : III, 1 (cr: 117-120)

Croteau, Jean-Philippe : I, 1 (cr : 75 78)

Danaux, Stéphanie : V, 2 (ar : 397 430)

Debien, Léon : V, 2 (ar : 345-395)

Demers, Frédéric : III, 2 (nr : 233 267)

De Vriendt, Patricia-Anne : V,1 (cr : 181-186)

Dionne, Patrick : III, 1 (cr : 111 116) ; III, 2 (cr : 279-284)

Doucet, Sophie : II, 1 (bib : 125-144)

Doyon, Nova : I, 2, (ar : 115-140) ; IV, 1 (cr: 111-119)

Drouin, Sébastien : V, 2 (ar : 241 276)

Ducharme, Michel : II, 1 (cr : 113 115) ; II, 2 (cr : 266-270) ; III, 1 (cr : 107-111) ; III, 2 (cr : 284 288) ;IV, 2 (cr : 335-340)

Facal, Cécile : V, 2 (ar : 215-239)

Fahrni, Magda : V, 1 (cr : 171-176)

Ferland, Catherine : V, 2 (cr : 514 518)
Foisy-Geoffroy, Dominique : I, 1 (ar : 51-68) ; III, 1 (éc : 61-95)

Gagnon, Nicole : IV, 2 (nc : 321-333)

Gaudreau, Guy : II, 1 (ar : 35-65)

Gélinas, Xavier : I, 2 (cr : 171-174) ; III, 2 (cr : 274-278) ; IV, 1 (cr : 145148)

Godin, Antoine : I, 2 (cr : 181-184)

Godin, Stéphanie : V, 1 (ar : 73-117)

Hare, John : V, 2 (ar : 307-344)

Hauser, Claude : V, 2 (cr : 518-520)

Hébert, Karine : II, 1 (bib : 125-144)

Huot, Giselle : IV, 2 (ar : 211-271)

Labelle, Gilles : III, 2 (ar : 193-231)

Lacroix, Benoît : IV, 1 (nc : 95-97)

Lamonde, Yvan : I, 2 (pers : 87-96)

Lapointe, Martine-Emmanuelle : III, 2 (cr : 269-273) ; V, 2 (cr : 509514)

Leroux, Éric : V, 2 (bib : 557-582)

Marcil, Olivier : II, 2 (ar : 193-231)

Marshall, Dominique : IV, 2 (cr : 344-348)

Melançon, François : V, 2 (ar : 277305)

Méthot, Mélanie : II, 1 (ar : 67-104)

Meunier, E.-Martin : III, 2 (ar : 149191)

Mills, Sean : III, 1 (cr : 102-107) ; IV, 2 (ar : 183-210) ; IV, 2 (cr : 340343)

Milot, David : V, 1 (cr : 186-194)

Ouellette, Mélanie : I, 1 (ar : 37-50)

Parmentier, Francis : V, 2 (cr : 537 556) 
Pépin, Elsa : V, 2 (ar : 465-496)

Perin, Roberto : IV, 1 (cr : 104-111)

Poirier, Patrick : IV, 1 (ar : 69-93)

Pomeyrols, Catherine : IV, 1 (bib : 159-176)

Rankin, Matthew : IV, 1 (cr : 149157)

Richard, Sacha : III, 1 (ar : 27-59)

Rouillard, Jacques : IV, 2 (cr : 352356)

Roy, Christian : IV, 2 (cr : 356-364) ; V, 1 (cr: 195-204)

Roy, Paul-Émile : II, 1 (pers : 1733)

Ruelland, Jacques G. : IV, 2 (cr : 348351)

Ryan, Pascale : IV, 1 (pers : 9-37) ; IV, 1 (cr : 130-136) ; V, 1 (cr: 167171)

Séguin, Renaud : II, 1 (cr: 115-123)
St-Pierre, Stéphane : V, 2 (cr : 532 537)

Tanguay, Daniel : III, 2 (ar : 129-147)

Thériault, Janine : V, 1 (ar : 7-71)

Thériault, Joseph Yvon : IV, 1 (cr : 99-103)

Tremblay, Micheline : II, 1 (ar : 3565)

Trépanier, Anne : V,1 (ar: 119-149) ; $\mathrm{V}, 2$ (cr: 521-525)

Trépanier, Pierre : II, 1 (pers : 5-15) ; II, 2 (cr : 261-265) ; IV, 2 (nc : 273 307)

Vannucci, Simone : V, 2 (ar : 431463)

Wallot, Jean-Pierre : V, 2 (ar : 307344)

Warren, Jean-Philippe : V, 2 (cr : 497-501) 


\section{INDEX DES SUJETS}

Académie de Montréal : I, 2 (115140)

Acadie : III, 1 (27-59)

Action catholique : IV, 2 (344-348) ; V, I (171-176)

Action française (Paris) : V, 2 (532537)

Aggiornamento: V, 1 (7-71)

Albala, Albert : IV, 2 (351-356)

Albert-Lévesque, Éditions : V, 2 (397-430)

Alliance laurentienne : III, 2 (274278)

Américanité : II, 1 (5-15) ; II, 1 (105112) ; V, I (151-161)

Anctil, Pierre : I, 1 (75-78)

Aquin, Hubert : III, 2 (269-273)

Archambault, Joseph-Alfred : I, 2 (97-113)

Archambault, Joseph-Papin : I, 2 (141-161)

Auzépy-Chavagnac, Véronique : $\mathrm{V}$, I (195-204)

Barbeau, Raymond : III, 2 (274-278)

Bernard, Harry : II, 1 (35-65)

Bibliographies : I, 2 (189-223) ; II, 1 (125-144) ; II, 2 (279-297); IV, 1 (159-176) ; V, 2 (557-582)

Bibliothèque : V, 2 (241-276); V, 2 (277-305) ; V, 2 (557-582)

Bienvenue, Louise : IV, 2 (344-348)

Boivin, Aurélien : V, 2 (509-514)
Bouchard, Catherine : IV, 1 (130136)

Bouchard, Gérard : I, 1 (75-78) ; II, 1 (105-112) ; IV, 2 (273-307, 309319)

Bourassa, Henri : I, 1 (69-74) ; III, 1 (97-102)

Bourget, Ignace : IV, 1 (104-111)

Boyer, Jean-Pierre : IV, 1 (111-119)

Brunet, Jean-Marc : III, 2 (274-278)

Bureau, Luc : V, 2 (518-520)

Canadianité : II, 1 (105-112)

Caouette, Réal : IV, 1 (145-148)

Cartésianisme : IV, 2 (349-351)

Catholicisme : I, 2 (141-161) ; IV, 1 (136-144) ; V, 1 (7-71)

Céline, Louis-Ferdinand : IV, 1 (3967)

Censure : V, 2 (241-276) ; V, 2 (465496) ; V, 2 (431-463)

Centre des femmes : IV, 2 (183-210)

Chauveau, Pierre-Joseph-Olivier : $\mathrm{V}, 2$ (525-531)

Clergé : II, 1 (5-15) ; II, 1 (17-33)

Coates, Collin M. : IV, 2 (340-343)

Commémoration : I, 2 (184-187); III, 1 (27-59) ; III, 1 (107-111)

Cooperative Commonwealth Federation: III, 1 (102-107) ; IV, 2(351-356)

Communisme et religion : I, 2 (141161) 
Conseil Supérieur : V, 2 (277-305)

Conservatisme : III, 2 (288-294)

Corbo, Claude : I, 2 (181-184); V, 2 (521-525)

Cornellier, Louis : V, I (181-186)

Corporatisme : I, 1 (51-68)

Courrier de St-Hyacinthe, Le : II, 1 (35-65)

Couture, Claude : II, 1 (113-115)

Crédit social : IV, 1 (145-148)

Critique littéraire : II, 1 (35-65) ; IV, 1 (39-67)

Culture : V, 1 (7-71)

Curtis, Bruce : III, 2 (284-288)

Dard, Olivier : IV, 2 (356-264)

Darwinisme : I, 2 (97-113)

Démocratie : I, 1 (37-50) ; IV, 1 (111119) ; V,I (151-161)

Denis, Mathieu : IV, 2 (352-356)

Déportation acadienne : III, 1 (2759)

Descartes, René : IV, 2 (349-351)

Dessaulles, Louis-Antoine : IV, 1 (104-111)

Djwa, Sandra : III, 1 (102-107)

Dominicains : V, 1 (7-71)

Drieu la Rochelle, Pierre : IV, 1 (3967)

Droit : IV, 2 (335-340) ; V, I (163166) ; V, 2 (277-305)

Du Calvet, Pierre : IV, 1 (111-119)

Dufresne, Jacques : III, 2 (279-284)

Dumont, Micheline : V, I (176-181)

Dumoulin, Olivier : IV, 1 (125-130)
École des beaux-arts de Montréal : IV, 2 (211-271)

École sociale populaire : I, 2 (141161)

Économie : I, 1 (51-68)

Édition littéraire : II, 2 (233-259), $\mathrm{V}, 2(397-430) ; \mathrm{V}, 2$ (431-463) ; V, 2 (307-344); V, 2 (557-582)

Éditions Mille Roches : II, 2 (233259)

Éducation : V, 2 (525-531)

État : III, 2 (284-288) ; IV, 2 (335340)

Etats-Unis : I, 1 (7-37) ; II, 1 (40), II, 2 (279-297)

Fédéralisme : I, 2 (163-169) ; V, 1 (73-117, 163-166)

Fabrègues, Jean de : V, I (195-204)

Féminisme : II, 1 (125-144) ; IV, 2 (183-210) ; V, 1 (73-117, 171-176, 176-181) ; V, 2 (465-496)

Foi et langue : I, 1 (7-37)

Franco-Américains : I, 1 (7-37)

France : IV, 1 (159-176)

Front de libération des femmes du Québec :IV, 2 (183-210)

Garneau, François-Xavier : III, 1 (9-26)

Gordon, Alan : III, 1 (107-111)

Grande Noirceur : III, 2 (149-191, 233-267)

Grand'Maison, Jacques : III, 2 (149191)

Greenwood, F. Murray : IV, 2 (335340) 
Groulx, Lionel : I, 1 (7-36) ; I, 2 (163169) ; IV, 2 (273-307, 309-319) ; V, 2 (502-508)

Harvey, Jean-Charles : I, 1 (78-80) ; II, 2 (155-192)

Hauser, Claude : V, I (167-171); V, 2 (532-537)

Helbronner, Jules : II, 1 (67-104)

Hétérodoxie : V, 2 (241-276)

Histoire : I, 1 (37-50)

Histoire - philosophie : III, 2 (193-231)

Histoire culturelle : IV, 1 (159-176)

Histoire intellectuelle - épistémologie : I, 1 (3-6) ; I, 2 (83-85, 87-96, 171-174) ; II, 1 (5-15) ; II, 2 (279-297) ; III, 2 (288-294) ; IV, 1 (9-37, 159-176) ; V, 2 (497-501)

Historien - rôle social : IV, 1 (125130)

Historiographie : I, 2 (87-96) ; II, 1 (105-112, 125-144) ; II, 2 (279297) ; III, 1 (9-26) ; III, 2 (233267) ; IV, 1 (9-37, 99-103, 159176) ; IV, 2 (273-307, 309-319) ; V, I (181-186)

Hitler, Adolf : IV, 1 (69-93)

Huot, Giselle : V, 2 (502-508)

Idées - histoire (voir « Histoire intellectuelle - épistémologie ")

Identité nationale : V, 1 (119-149, 151-161)

Impérialisme : III, 1 (97-102)

Imprimerie : V, 2 (307-344) ; V, 2 (557-582)

Index : V, 2 (241-276)
Institut canadien : IV, 1 (104-111)

Institut d'histoire de l'Amérique française : I, 2 (174-180) ; II, 2 (271-277)

Jansénisme : V, 2 (241-276, 514-518)

Jautard, Valentin : I, 2 (115-140)

Jésuites : V, 1 (7-71) ; V, 2 (431-463)

Jeune droite (France) : IV, 2 (356264) ; V, I (195-204)

Jeunesse ouvrière catholique : V, I (171-176)

Journaux étudiants : V, 2 (345-395)

Jura (Suisse) : V, I (167-171)

Justice : IV, 1 (111-119) ; IV, 2 (335340)

Kelly, Stéphane : II, 2 (266-270) ;IV, 1 (99-103)

Labrecque, Jean-Claude :IV, 1 (149157)

Lachance, Louis : I, 2 (163-169) ;II, 2 (155-192)

Lacombe, Sylvie : III, 1 (97-102)

Laflèche, Guy : V, 2 (514-518)

Lalonde-Rémillard, Juliette : V, 2 (502-508)

Lamarckisme : I, 2 (97-113)

Lamonde, Yvan : I, 2 (171-174, 181184) ; II, 1 (113-115) ; V, I (167171) ; V, 2 (497-501, 537-556)

Langue et foi : I, 1 (7-37)

Langue - question linguistique : II, 2 (193-231)

La Patrie : IV, 1 (69-93)

La Presse : II, 1 (67-104)

Laurendeau, Albert : I, 2 (97-113) 
Laurier, Wilfrid : II, 2 (266-270)

Lavertu, Yves : I, 1 (78-80)

Leclerc, Gilles : III, 2 (193-231)

Lecture : V, 2 (215-239, 465-496, 431$463,537-556,557-582)$

Le Devoir : II, 2 (193-231)

Le Droit : II, 1 (35-65)

Libéralisme : I, 1 (37-50) ; I, 2 (141161) ; II, 2 (193-231) ; IV, 1 (3967, 69-93, 136-144)

Littérature : I, 2 (115-140) ; II, 1 (1733) ; II, 2 (233-259) ; IV, 1 (3967) ; V, 2 (509-514)

Livre : V, 2 (215-239, 241-276, 277 $305,307-344,431-463,465-496$, 537-556, 557-582)

Lüsebrink, Hans-Jürgen : IV,1 (119124)

Macdonald, John Alexander : II, 2 (266-270)

Mackenzie King, William Lyon : II, 2 (266-270)

Maintenant: V, 1 (7-71)

Marcil, Claude : IV, 1 (145-148)

Marcil, Olivier : IV, 1 (136-144)

Marion, Jean-Claude : IV, 1 (145148)

Massolin, Philip : III, 2 (288-294)

Médecine : I, 2 (97-113)

Mesplet, Fleury : I, 2 (115-140)

Minville, Esdras : I, 1 (51-68)

Miron, Gaston : III, 2 (269-273)

Modernité : IV, 1 (69-93) ; V, I (151161)

Montreuil, Sophie : V, 2 (537-556)
Morgan, Cecilia : IV, 2 (340-343)

Morin, Jacques-Victor : IV, 2 (351356)

Narration : III, 1 (9-26)

Nation - définition : I, 1 (7-37, 5168) ; II, 2 (155-192) ; IV, 1 (130136)

Nationalisme : I, 1 (7-37, 51-68, 6974) ; I, 2 (141-161 ; 163-169) ; II, 1 (5-15) ; II, 2 (155-192, 193-231); III, 1 (61-95, 97-102) ; IV, 1 (130136, 136-144) ; IV, 2 (183-210, 273-307, 309-319) ; V, I (186-194)

Nation-Building: I, 2 (184-187)

Nazisme : IV, 1 (69-93)

Nelles, Henry Vivian : I, 2 (184187)

Nevers, Edmond de : IV, 1 (119-124)

Non-conformisme (France) : IV, 2 (356-264) ; V, I (195-204)

Nouveau-Brunswick : III, 1 (27-59)

Nouveau parti démocratique : III, 1 (102-107) ; IV, 2 (351-356)

Nouvelle sensibilité historique : IV, 1 (99-103)

Ouvriers (voir " Travailleurs")

Pâquet, Louis-Adolphe : III, 1 (6195)

Parent, Étienne : II, 1 (113-115)

Parti québécois : IV, 2 (183-210)

Patriotisme (voir " Nationalisme ")

Peinture : IV, 2 (211-271)

Personnalisme : IV, 2 (356-264); V, I (195-204) 
Philosophie : II, 2 (155-192) ; III, 2 (279-284) ; IV, 1 (95-97) ; IV, 2 (349-351)

Piché, Lucie : V,I (171-176)

Pleau, Jean-Christian : III, 2 (269273)

Poésie : IV, 1 (95-97)

Politique : I, 1 (37-50)

Pomeyrols, Catherine : IV, 1 (159176) ; V, 2 (532-537)

Presse - histoire : I, 2 (115-140) ; II, 1 (35-65) ; II, 2 (193-231) ; IV, 1 (39-67, 69-93) ; V, 2 (307-344, 345-395)

Protestantisme: V, 2 (241-276)

Provost, Roland : I, 1 (69-74)

Québécoises deboutte!: IV, 2 (183210)

Racisme : II, 2 (155-192) ;

Radio-Canada : III, 2 (233-267)

Rassemblement pour l'indépendance nationale : IV, 1 (149-157)

Raymond, Jean-François de : IV, 2 (349-351)

Rébellions : IV, 2 (335-340)

Récits de voyage : V, 1 (119-149); V, 2 (518-520)

Référendum (1980) : V, 1 (73-117)

Réformisme : I, 1 (51-68) ; I, 2 (141161) ; III, 2 (149-191)

Régionalisme - économie : I, 1 (51-68)

Régionalisme - littérature : II, 1 (35-65)

Relations : V, 1 (7-71)
Religion : I, 1 (7-36 ; 37-50) ; I, 2 (97-113, 141-161, 163-169);II, 2 (155-192) ; III, 2 (149-191) ; V, 2 (241-276)

Renaissance acadienne : III, 1 (2759)

Révisionnisme : II, 1 (105-112)

Révolution tranquille : II, 1 (1733) ; III, 2 (129-147) ; III, 2 (149191) ; III, 2 (193-231) ; III, 2 (233267) ; III, 2 (269-273)

Revues : V, 1 (7-71) ; V, 2 (431-463)

Robillard, Edmond : III, 1 (111-16)

Robinson, Ira : I, 1 (75-78)

Ryan, Claude : II, 2 (193-231) ; IV, 1 (136-144)

Sabourin, Hélène : V, 2 (525-531)

Saywell, John T. : V, I (163-166)

Science : I, 2 (97-113)

Scott, Frank : III, 1 (102-107)

Secord, Laura : IV, 2 (340-343)

Séminaire de Québec : V, 2 (241276)

Sensibilité historique : IV, 1 (99103)

Sentinellisme : I, 1 (7-37)

Socialisme : IV, 2 (183-210) ; V, I (186-194)

Sociologie : IV, 2 (321-333)

Statistique : III, 2 (284-288)

Saint-Denys Garneau, Hector de : IV, 2 (211-271)

Suisse romande : V, I (167-171)

Syndicalisme : IV, 2 (351-356)

Télévision : III, 2 (233-267) 
Thériault, Joseph Yvon : V, I (151- Vadeboncœur, Pierre : III, 2 (129161) 147)

Thério, Adrien : IV, 1 (104-111) Vallières, Pierre : V, I (186-194)

Thomisme : I, 2 (163-169) ; II, 1 (5- Vatican II : V, 1 (7-71)

Verchères, Madeleine de : IV, 2 (340-

Toqueville, Alexis de : V, 1 (119 149) ; V, 2 (521-525)

Toupin, Louise : V, I (176-181)

Travailleurs : II, 1 (67-104) 343)

Viatte, Auguste : II, 2 (261-265) ; V, I (167-171)

Trépanier, Pierre : V, 2 (502-508) Warren, Jean-Philippe : IV, 2 (321-

Trudeau, Pierre Elliott : I, 1 (3750) ; II, 2 (266-270) 333)

Trudel, Serge $: \mathrm{V}, 2(514-518)$ Wright, Barry : IV, 2 (335-340) Yvettes : V, 1 (73-117) 